\title{
Article
}

\section{Null Homology Groups and Stable Currents in Warped Product Submanifolds of Euclidean Spaces}

\author{
Yanlin Li ${ }^{1,+}$, Pişcoran Laurian-Ioan ${ }^{2, *,+}\left(\mathbb{D}\right.$, Akram Ali $^{3,+}$ and Ali H. Alkhaldi ${ }^{3,+}$ \\ 1 School of Mathematics, Hangzhou Normal University, Hangzhou 311121, China; liyl@hznu.edu.cn \\ 2 Department of Mathematics and Computer Science Victoriei 76, North University Center of Baia Mare \\ Technical University of Cluj Napoca, 430122 Baia Mare, Romania \\ 3 Department of Mathematics, College of Science, King Khalid University, Abha 61413, Saudi Arabia; \\ akali@kku.edu.sa (A.A.); ahalkhaldi@kku.edu.sa (A.H.A.) \\ * Correspondence: Laurian.PISCORAN@mi.utcluj.ro \\ + These authors contributed equally to this work.
}

Citation: Li, Y.; Laurian-Ioan, P.; Ali, A.; Alkhaldi, A.H. Null Homology Groups and Stable Currents in Warped Product Submanifolds of Euclidean Spaces. Symmetry 2021, 13, 1587. https://doi.org/10.3390/ sym13091587

Academic Editor: George Kaimakamis

Received: 30 June 2021

Accepted: 19 August 2021

Published: 28 August 2021

Publisher's Note: MDPI stays neutral with regard to jurisdictional claims in published maps and institutional affiliations.

Copyright: (C) 2021 by the authors. Licensee MDPI, Basel, Switzerland. This article is an open access article distributed under the terms and conditions of the Creative Commons Attribution (CC BY) license (https:// creativecommons.org/licenses/by/ $4.0 /)$.

\begin{abstract}
In this paper, we prove that, for compact warped product submanifolds $M^{n}$ in an Euclidean space $\mathbb{E}^{n+k}$, there are no stable $p$-currents, homology groups are vanishing, and $M^{3}$ is homotopic to the Euclidean sphere $\mathbb{S}^{3}$ under various extrinsic restrictions, involving the eigenvalue of the warped function, integral Ricci curvature, and the Hessian tensor. The results in this paper can be considered an extension of Xin's work in the framework of a compact warped product submanifold, when the base manifold is minimal in ambient manifolds.
\end{abstract}

Keywords: warped product submanifolds; euclidean spaces; homology group; homotopic; fundamental groups; stable currents

\section{Introduction and Preliminaries}

The geometric structure and topological properties of submanifolds in different ambient spaces have been studied on a large scale during the past few years. Many results have shown that there is a close relationship between the non-existence of stable currents and the vanishing homology groups of submanifolds in a different class of ambient manifolds by using the pinching condition of the second fundamental form [1]. In [1], Lawson and Simons provided the optimization of the second fundamental form, which forces homology to vanish in a range of intermediate dimensions, and also the nonexistence of stable currents in submanifolds of simply connected space form. They obtained the following theorem, which is the key result of that paper.

Theorem $\mathbf{1}([1,2])$. Let $M^{n}$ be a compact $n$-dimensional submanifold of the space form $\tilde{M}(c)$ of constant curvature $c \geq 0$ and the second fundamental form $\mathbf{B}$, and $p, q$ are any positive integers such that $p+q=n$. If the inequality

$$
\sum_{\alpha=1}^{p} \sum_{\beta=p+1}^{n}\left(2\left\|\mathbf{B}\left(e_{\alpha}, e_{\beta}\right)\right\|^{2}-g\left(\mathbf{B}\left(e_{\alpha}, e_{\alpha}\right), \mathbf{B}\left(e_{\beta}, e_{\beta}\right)\right)\right)<p q c
$$

is satisfied, then there are no stable p-currents on $M^{n}$ and

$$
H_{p}\left(M^{n}, \mathbb{Z}\right)=H_{q}\left(M^{n}, \mathbb{Z}\right)=0
$$

for any $p \in M^{n}$ and an orthonormal frame $\left\{e_{i}\right\}_{1<i \leq n}$ of tangent space $T M^{n}$, where $H_{i}\left(M^{n}, \mathbb{Z}\right)$ is the $i$-th homology group of $M^{n}$ with integer coefficients.

As an application of Ricci curvature, Vlachos [3] proved the following result due to Theorem 1. 
Theorem 2 ([3]). Let $M^{n}$ be a compact oriented $n$-dimensional submanifold of $(n+k)$-dimensional Euclidean space $\mathbb{E}^{n+k}$ and assume that, for each unit vector $X$, the Ricci curvature is satisfied:

$$
\operatorname{Ric}(X)>\delta_{1}(n) g\left(A_{H} X, X\right),
$$

where $A_{H}$ is the shape operator with respect to the mean curvature $H$, and $\delta_{1}(n)$ is a constant given by $\delta_{1}(n)=n(n-3) /(n-1)$ if $n$ is odd and $\delta_{1}(n)=n-2$ if $n$ is even. Then there are no stable currents in $M^{n}$. Moreover, $M^{n}$ is homeomorphic to $\mathbb{S}^{n}$.

To show that the result (2) is optimal, we pay attention to a minimal submanifold of $\mathbb{C} \mathbb{P}^{n}$ of the constant holomorphic sectional curvature $\frac{2 n}{n+1}$ into $\mathbb{S}^{n(n+2)-1}$, that was constructed in [4] by Wallah. Furthermore, if $n=2$, we have $\phi_{n}: \mathbb{C P}^{n} \longrightarrow \mathbb{E}^{n(n+2)}$ satisfies $\operatorname{Ric}(X)=\delta_{1}(2 n) g\left(A_{H} X, X\right)$ for each unit tangential vector $X$ [3]. On the other hand, $\mathbb{C P}^{2}$ is not homeomorphic to $\mathbb{S}^{4}$ and this means that the pinching condition (2) is optimal. Moreover, using Theorem 1 in [5], it was proved that if compact oriented $n$ dimensional submanifold of $(n+m)$-dim space form $\mathbb{F}_{c}^{n+k}$ satisfies the second fundamental form pinching condition $S<a(n, k,|H|, c)$, for any integral number $k$ such that $0<k<n$, the $p$-th homology groups are vanishing, $H_{p}\left(M^{n}, \mathbb{Z}\right)=0$, for all $p \in\{k, \cdots, n-k\}$, and if the fundamental group $\pi_{1}\left(M^{n}\right)$ is finite and simply connected, $M^{n}$ is homeomorphic to $\mathbb{S}^{n}$. Using Theorem 1, Xu and Gu [6] extended the pinching condition in terms of the Ricci curvature. They also showed that, for a compact submanifold $M^{n}$ in space form $\mathbb{F}_{c}^{n+k}$, if it satisfies Ric $>(n-2)\left(c+H^{2}\right)$ then $M^{n}$ is homeomorphic to $\mathbb{S}^{n}$, where $H$ is the mean curvature of $M^{n}$ and $c$ is a constant sectional curvature. Motivated by the non-existence of stable currents or stable submanifolds, a number of topological properties have been studied in [2,5,7-14] by using Theorem 1 .

Inspired by the above-mentioned aspects, we want to obtain some similar results for warped product submanifold theory where the second fundamental form pinching condition will be replaced by the warping function. It is interesting and meaningful to know how the topological and geometrical of warped product submanifolds is affected by the main intrinsic invariant and main extrinsic curvature invariant. Taking advantage of the Nash embedding theorem [15] and Nolker [16], we will prove the nonexistence stable integral $p$-currents in a compact warped product submanifold in a flat space or a Euclidean space $\mathbb{E}^{n+k}$. We provide some basic notions needed in this paper.

Let $M^{n}$ be isometrically immersed into a Riemannian manifold $\widetilde{M}$ with induced metric g. If $\nabla$ and $\nabla^{\perp}$ are the induced Riemannian connections on the tangent bundle $T M$ and the normal bundle $T^{\perp} M$ over $M^{n}$, respectively, then the Gauss and Weingarten formulas are given by

$$
\begin{array}{r}
\widetilde{\nabla}_{U} V=\nabla_{U} V+\mathbf{B}(U, V), \\
\widetilde{\nabla}_{U} \xi=-A_{\xi} U+\nabla_{U}^{\perp} \xi
\end{array}
$$

for each $U, V \in \mathfrak{X}(T M)$ and $\xi \in \mathfrak{X}\left(T^{\perp} M\right)$, where $\mathbf{B}$ and $A_{\xi}$ are the second fundamental form and the shape operator (corresponding to the normal vector field $\xi$ ), respectively [17]. They are related as follows: $g(\mathbf{B}(U, V), \xi)=g\left(A_{\tilde{\xi}} U, V\right)$, where $g$ denotes the Riemannian metric on $\widetilde{M}$ as well as the metric induced on $M^{n}$. The Gauss equation for a submanifold $M$ is given by:

$$
\begin{aligned}
\widetilde{R}\left(X_{1}, Y_{1}, Z_{1}, W_{1}\right)= & R\left(X_{1}, Y_{1}, Z_{1}, W_{1}\right)+g\left(\mathbf{B}\left(X_{1}, Z_{1}\right), \mathbf{B}\left(Y_{1}, W_{1}\right)\right) \\
& -g\left(\mathbf{B}\left(X_{1}, W_{1}\right), \mathbf{B}\left(Y_{1}, Z_{1}\right)\right),
\end{aligned}
$$

for $X_{1}, Y_{1}, Z_{1}, W_{1} \in \mathfrak{X}(T M)$, where $\widetilde{R}$ and $R$ are the curvature tensors on $\widetilde{M}$ and $M^{n}$, respectively [17]. In $\widetilde{M}$, we choose a local orthonormal frame $\left\{e_{1}, e_{2}, \cdots, e_{n}, e_{n+1}, \cdots, e_{n+k}\right\}$. 
The mean curvature vector $H$ for a local orthonormal frame $\left\{e_{1}, e_{2}, \cdots, e_{n}\right\}$ on $M^{n}$ is defined by

$$
\|\mathbf{H}\|^{2}=\frac{1}{n^{2}} \sum_{r=n+1}^{n+k}\left(\sum_{i=1}^{n} \mathbf{B}_{i i}^{r}\right)
$$

In addition, we set

$$
\mathbf{B}_{i j}^{r}=g\left(\mathbf{B}\left(e_{i}, e_{j}\right), e_{r}\right), \quad 1 \leq i, j \leq n \& n+1 \leq r \leq n+k .
$$

And we give another Riemannian intrinsic invariant's definition named the scalar curvature of $M^{n}$, we denoted by $\tau\left(T_{x} M^{n}\right)$, at some $x$ in $M^{n}$, is given by the following:

$$
\tau\left(T_{x} M^{n}\right)=\sum_{1 \leq \alpha<\beta \leq n} K_{\alpha \beta},
$$

where $K_{\alpha \beta}=K\left(e_{\alpha} \wedge e_{\beta}\right)$. The (8) is equivalent to below equation, which will be frequently used in subsequent proofs:

$$
2 \tau\left(T_{x} M^{n}\right)=\sum_{1 \leq \alpha<\beta \leq n} K_{\alpha \beta}, 1 \leq \alpha, \beta \leq n .
$$

Similarly, the scalar curvature $\tau\left(L_{x}\right)$ of an $L$-plane is provided by the following:

$$
\tau\left(L_{x}\right)=\sum_{1 \leq \alpha<\beta \leq n} K_{\alpha \beta} .
$$

If plane sections are spanned by $e_{\alpha}$ and $e_{\beta}$ at $x$, sectional curvature of $M^{n}$ and $\widetilde{M}^{n+k}$ are denoted by $K_{\alpha \beta}$ and $\widetilde{K}_{\alpha \beta}$, respectively. Thus, $K_{\alpha \beta}$ and $\widetilde{K}_{\alpha \beta}$ are the intrinsic and extrinsic sectional curvatures of the span $\left\{e_{\alpha}, e_{\beta}\right\}$ at $x$ [18]. Taking advantage of Gauss Equations (5) and (8), we have

$$
\sum_{1 \leq \alpha<\beta \leq n+k} K_{\alpha \beta}=\sum_{1 \leq \alpha<\beta \leq n+k} \widetilde{K}_{\alpha \beta}+\sum_{r=n+1}^{n+k}\left(\mathbf{B}_{\alpha \alpha}^{r} \mathbf{B}_{\beta \beta}^{r}-\left(\mathbf{B}_{\alpha \beta}^{r}\right)^{2}\right) .
$$

On the other hand, the idea of warped product manifolds was originally introduced by Bishop and O'Neill [19] for manifolds of negative curvature. Assume that $N_{1}^{p}$ and $N_{2}^{q}$ are two Riemannian manifolds with their Riemannain metrics $g_{1}$ and $g_{2}$, respectively. Let $f$ be a smooth function defined on $N_{1}^{p}$. A warped product manifold is $M^{n}=N_{1}^{p} \times{ }_{f} N_{2}^{q}$ with $n=p+q$ and the Riemannian metric $g=g_{1}+f^{2} g_{2}$. Assume that $M^{n}=N_{1}^{p} \times{ }_{f} N_{2}^{q}$ is a warped product manifold, then for any $X \in \Gamma\left(T N_{1}\right)$ and $Z \in \Gamma\left(T N_{2}\right)$, we find that

$$
\nabla_{Z} X=\nabla_{X} Z=(X \ln f) Z \text {. }
$$

Thus, the function $f$ is called a warping function on $M^{n}$. The following lemma is a direct consequence of the warped product manifolds:

Lemma 1 ([19]). Let $M=N_{1} \times_{f} N_{2}$ be a warped product manifold. Then we have

(i) $\nabla_{Z} X=\nabla_{X} Z=\frac{(X f)}{f} Z$,

(ii) $\nabla_{Z} W=\nabla_{Z}^{\prime} W-g(Z, W) \nabla \ln f$,

for any $X, Y \in \mathfrak{X}\left(T N_{1}\right)$ and $Z, W \in \mathfrak{X}\left(T N_{2}\right)$, where $\nabla$ and $\nabla^{\prime}$ denote the Levi-Civita connections on $M$ and $N_{2}$, respectively. Further, $\nabla(\ln f)$ is the gradient of $\ln f$, given by

$$
g(\nabla \ln f, X)=X(\ln f) .
$$


Thus, by using (i) of Lemma 1, we have

$$
R(X, Z) Y=\frac{H^{f}(X, Y)}{f} Z,
$$

where $H^{f}$ is a Hessian tensor of $f$. Let $\left\{e_{1}, \ldots, e_{n}\right\}$ be an local orthonormal frame of vector field $M^{n}$, thus the squared norm of the gradient of the positive differentiable function $\varphi$ for an orthonomal frame $\left\{e_{1}, \ldots, e_{n}\right\}$ is defined as:

$$
\|\nabla \varphi\|^{2}=\sum_{i=1}^{n}\left(e_{i}(\varphi)\right)^{2}
$$

Let $\varphi$ be a differential function defined on $M^{n}$. The gradient $\vec{\nabla} \varphi$ is given by:

$$
g(\vec{\nabla} \varphi, X)=X \varphi, \text { and } \vec{\nabla} \varphi=\sum_{i=1}^{n} e_{i}(\varphi) e_{i}
$$

and the Laplacian $\Delta \varphi$ of $\varphi$ is defined as:

$$
\begin{aligned}
\Delta \varphi & =-\sum_{i=1}^{n}\left\{\left(\nabla_{e_{i}} e_{i}\right) \varphi-e_{i}\left(e_{i}(\varphi)\right)\right\} \\
& =\sum_{i=1}^{n} g\left(\nabla_{e_{i}} \operatorname{grad} \varphi, e_{i}\right)=\operatorname{trHess}(\varphi) .
\end{aligned}
$$

Remark 1. It should be noted that we consider the opposite sign of Chen [20] of the Laplacian of the function $\varphi$, that is $\Delta=\frac{d}{d t}$ on the real line

Moreover, because the units vector fields $X$ and $Z$ are tangent to $N_{1}^{p}, N_{2}^{q}$, respectively, we get:

$$
\begin{aligned}
K(X \wedge Z) & =g(R(X, Z) X, Z)=\left(\nabla_{X} X\right) \ln f g(Z, Z)-g\left(\nabla_{X}((X \ln f) Z), Z\right) \\
& =\left(\nabla_{X} X\right) \ln f g(Z, Z)-g\left(\nabla_{X}(X \ln f) Z+(X \ln f) \nabla_{X} Z, Z\right) \\
& =\left(\nabla_{X} X\right) \ln f g(Z, Z)-(X \ln f)^{2}-X(X \ln f)
\end{aligned}
$$

Suppose that $\left\{e_{1}, \cdots e_{n}\right\}$ is an orthonormal frame for $M^{n}$, then summing up over the vector fields such that

$$
\sum_{i=1}^{p} \sum_{j=p+1}^{n} K\left(e_{i} \wedge e_{j}\right)=\sum_{i=1}^{p} \sum_{j=p+1}^{n}\left(\left(\nabla_{e_{i}} e_{i}\right) \ln f-e_{i}\left(e_{i} \ln f\right)-\left(e_{i} \ln f\right)^{2}\right)
$$

it implies that

$$
\sum_{i=1}^{p} \sum_{j=p+1}^{n} K\left(e_{i} \wedge e_{j}\right)=-\frac{q \Delta f}{f}
$$

The following remarks are consequences of Lemma 1:

Remark 2. A warped product manifold $M^{n}=N_{1}^{p} \times_{f} N_{2}^{q}$ is said to be trivial or simply a Riemannian product manifold if the warping function $f$ is constant.

Remark 3. If $M^{n}=N_{1}^{p} \times_{f} N_{2}^{q}$ is a warped product manifold, then $N_{1}^{p}$ is totally geodesic and $N_{2}^{q}$ is a totally umbilical submanifold of $M^{n}$, respectively. 


\section{Main Results}

In this section, we present the main results of this paper.

Theorem 3. Let $\varphi: M^{p+q}=N_{1}^{p} \times_{f} N_{2}^{q} \longrightarrow \mathbb{E}^{p+q+k}$ be a $N_{1}^{p}$-minimal isometric immersion from a compact warped product submanifold $M^{p+q}$ into an $(p+q+k)$-dimensional Euclidean space $\mathbb{E}^{p+q+k}$, satisfying the following inequality

$$
f \Delta f<2 q\|\nabla f\|^{2},
$$

where $\Delta f$ and $\nabla f$ are the Laplacian and the gradient of the warping function $f$. Then, $M^{p+q}$ satisfies the following:

(i) There do not exist stable $p$-currents in $M^{p+q}$ and

$$
H_{p}\left(M^{p+q}, \mathbb{Z}\right)=H_{q}\left(M^{p+q}, \mathbb{Z}\right)=0
$$

for all integers $p, q$ such that $p+q=n$, where $H_{i}\left(M^{p+q}, \mathbb{Z}\right)$ is the $i$-th homology groups of $M^{n}$ with integer coefficients.

(ii) The fundamental group $\pi_{1}\left(M^{p+q}\right)$ is vanished, that is, $\pi_{1}\left(M^{p+q}\right)=0$.

(iii) If $p+q=3$, then $M^{3}$ is homotopic to a Euclidean sphere $\mathbb{S}^{3}$.

Motivated by the geometric rigidity Theorem 3, the second aim of this paper is to prove a new topological theorem for compact warped product submanifolds in terms of the Ricci curvature and using the eigenvalues of the Laplacian of the warping function. In particular, we can give the following topological sphere theorem:

Theorem 4. Let $\varphi: M^{p+q}=N_{1}^{p} \times_{f} N_{2}^{q} \longrightarrow \mathbb{E}^{p+q+k}$ be a $N_{1}^{p}$-minimal isometric immersion from a compact warped product submanifold $M^{p+q}$ into an $(p+q+k)$-dimensional Euclidean space $\mathbb{E}^{p+q+k}$ and the warping function $f$ is an eigenfunction of the Laplacian of $M^{n}$ associated to the first positive eigenvalue $\lambda_{1}$ satisfying the following inequality:

$$
\int_{M^{n}}\left\{\left\|\nabla^{2} f\right\|^{2}+\operatorname{Ric}(\nabla f, \nabla f)\right\} d V>\left(\frac{\lambda_{1}^{2}}{2 q}\right)\left(-\int_{M^{n}} f^{2} d V\right),
$$

for integral Hessian tensor $\nabla^{2} f$ of the warping function $f$ and integral Ricci curvature along the gradient $\nabla f$. Then, $M^{p+q}$ satisfies the following:

(i) There do not exist stable $p$-currents in $M^{p+q}$ and

$$
H_{p}\left(M^{p+q}, \mathbb{Z}\right)=H_{q}\left(M^{p+q}, \mathbb{Z}\right)=0
$$

for all integers $p, q$ such that $p+q=n$, where $H_{i}\left(M^{p+q}, \mathbb{Z}\right)$ is the $i$-th homology groups of $M^{n}$ with integer coefficients.

(ii) The fundamental group $\pi_{1}\left(M^{p+q}\right)$ is vanished, that is, $\pi_{1}\left(M^{p+q}\right)=0$.

(iii) If $p+q=3$, then $M^{3}$ is homotopic to a Euclidean sphere $\mathbb{S}^{3}$.

As an immediate application of Theorem 4, we can now give the following:

Corollary 1. Assume that $\varphi: M^{p+q}=N_{1}^{p} \times_{f} N_{2}^{q} \longrightarrow \mathbb{E}^{p+q+k}$ is an $N_{1}^{p}$-minimal isometric immersion from a compact warped product submanifold $M^{p+q}$ into $a(p+q+k)$-dimensional Euclidean space $\mathbb{E}^{p+q+k}$ satisfying the following inequality:

$$
\int_{M^{n}} \sum_{i=1}^{p}\left\|\mathbf{B}\left(\nabla f, e_{i}\right)\right\|^{2} d V<\int_{M^{n}}\left\{\left\|\nabla^{2} f\right\|^{2}+\frac{(\Delta f)^{2}}{2 q}\right\} d V
$$

Then, $M^{p+q}$ satisfies the following: 
(i) There do not exist stable p-currents in $M^{p+q}$ and

$$
H_{p}\left(M^{p+q}, \mathbb{Z}\right)=H_{q}\left(M^{p+q}, \mathbb{Z}\right)=0,
$$

for all integers $p, q$ such that $p+q=n$, where $H_{i}\left(M^{p+q}, \mathbb{Z}\right)$ is the $i$-th homology groups of $M^{n}$ with integer coefficients.

(ii) The fundamental group $\pi_{1}\left(M^{p+q}\right)$ is vanished, that is, $\pi_{1}\left(M^{p+q}\right)=0$.

(iii) If $p+q=3$, then $M^{3}$ is homotopic to a Euclidean sphere $\mathbb{S}^{3}$.

Another interesting result, which is a direct consequence of Corollary 1 , is the following:

Corollary 2. Assume that $\varphi: M^{p+q}=N_{1}^{p} \times_{f} N_{2}^{q} \longrightarrow \mathbb{E}^{p+q+k}$ is an $N_{1}^{p}$-minimal isometric immersion from a compact warped product submanifold $M^{p+q}$ into $a(p+q+k)$-dimensional Euclidean space $\mathbb{E}^{p+q+k}$ such that $\nabla f \in \operatorname{Ker} \mathbf{B}$ and satisfies the following:

$$
\int_{M^{n}}\left\{\left\|\nabla^{2} f\right\|^{2}+\frac{(\Delta f)^{2}}{2 q}\right\} d V>0,
$$

where $\nabla^{2} f$ is the Hessian of the warped function $f$. Then, $M^{p+q}$ holds the following:

(i) There do not exist stable p-currents in $M^{p+q}$ and

$$
H_{p}\left(M^{p+q}, \mathbb{Z}\right)=H_{q}\left(M^{p+q}, \mathbb{Z}\right)=0,
$$

for all integers $p, q$ such that $p+q=n$, where $H_{i}\left(M^{p+q}, \mathbb{Z}\right)$ is the $i$-th homology groups of $M^{n}$ with integer coefficients.

(ii) The fundamental group $\pi_{1}\left(M^{p+q}\right)$ is vanished, that is, $\pi_{1}\left(M^{p+q}\right)=0$.

(iii) If $p+q=3$, then $M^{3}$ is homotopic to a Euclidean sphere $\mathbb{S}^{3}$.

Here we consider that the base of the warped product submanifold is Ricci-flat and we find the results below as a corollary of Theorem 4:

Corollary 3. Let $\varphi: M^{p+q}=N_{1}^{p} \times_{f} N_{2}^{q} \longrightarrow \mathbb{E}^{p+q+k}$ be an $N_{1}^{p}$-minimal isometric immersion from a compact warped product submanifold into a Euclidean space $\mathbb{E}^{p+q+k}$ with base manifold $N_{1}^{p}$ is Ricci flat and satisfies the following:

$$
\int_{M^{n}} f \Delta f d V<\left(\frac{2 q}{\lambda_{1}}\right) \int_{M^{n}}\left\|\nabla^{2} f\right\|^{2} d V,
$$

where $\lambda_{1}$ is an eigenvalue of the warped function $f$. Then, $M^{p+q}$ holds the following:

(i) There do not exist stable $p$-currents in $M^{p+q}$ and

$$
H_{p}\left(M^{p+q}, \mathbb{Z}\right)=H_{q}\left(M^{p+q}, \mathbb{Z}\right)=0,
$$

for all integers $p, q$ such that $p+q=n$, where $H_{i}\left(M^{p+q}, \mathbb{Z}\right)$ is the $i$-th homology groups of $M^{n}$ with integer coefficients.

(ii) The fundamental groups $\pi_{1}\left(M^{p+q}\right)$ is vanished, that is, $\pi_{1}\left(M^{p+q}\right)=0$.

(iii) If $p+q=3$, then $M^{3}$ is homotopic to a Euclidean sphere $\mathbb{S}^{3}$.

Theorem 3 is a main topological sphere theorem for a compact warped product submanifold without the assumption that $M^{n}$ is simply connected. Moreover, our result becomes more significant due to involving the new pinching conditions in terms of the warping function, integral of the squared norm of the Hessian tensor, integral Ricci curvature and the first non-zero eigenvalue of the warped function [21]. And we could find out more about the applications and meanings of physics in some articles [22-26] about these topics. From the perspective of applied physics, the main results of this paper have great physical significance. 


\section{Proof of Main Results}

In the first case, we assume that the warped product submanifold isometrically immersed in a Euclidean $\mathbb{E}^{n+k}$ with codimension $k$ and this proves the result.

\subsection{Proof of Theorem 3}

Let $\operatorname{dim}\left(N_{1}\right)=p$ and $\operatorname{dim}\left(N_{2}\right)=q$ and consider $\left\{e_{1}, e_{2}, \cdots, e_{p}\right\}$, and $\left\{e_{p+1}^{*}, \cdots, e_{n}^{*}\right\}$ to be orthonormal frames of $T N_{1}$ and $T N_{2}$, respectively. Then, from Gauss Equation (5) for a Euclidean space $\mathbb{E}^{n+k}$, we have

$$
\begin{aligned}
\sum_{\alpha=1}^{p} \sum_{\beta=p+1}^{n}\left\{2\left\|\mathbf{B}\left(e_{\alpha}, e_{\beta}\right)\right\|^{2}\right. & \left.-g\left(\mathbf{B}\left(e_{\alpha}, e_{\alpha}\right), \mathbf{B}\left(e_{\beta}, e_{\beta}\right)\right)\right\} \\
= & \sum_{r=n+1}^{n+k} \sum_{\alpha=1}^{p} \sum_{\beta=p+1}^{n}\left(\mathbf{B}_{\alpha \beta}^{r}\right)^{2} \\
& +\sum_{\alpha=1}^{p} \sum_{\beta=p+1}^{n}\left\{\left\|\mathbf{B}\left(e_{\alpha}, e_{\beta}\right)\right\|^{2}-g\left(\mathbf{B}\left(e_{\alpha}, e_{\beta}\right), \mathbf{B}\left(e_{\alpha}, e_{\beta}\right)\right)\right\} .
\end{aligned}
$$

From Gauss Equation (5) for the Euclidean space $\mathbb{E}^{n+k}$, we get further

$$
\begin{aligned}
\sum_{\alpha=1}^{p} \sum_{\beta=p+1}^{n}\left\{2\left\|\mathbf{B}\left(e_{\alpha}, e_{\beta}\right)\right\|^{2}-\right. & \left.g\left(\mathbf{B}\left(e_{\alpha}, e_{\alpha}\right), \mathbf{B}\left(e_{\beta}, e_{\beta}\right)\right)\right\} \\
= & \sum_{r=n+1}^{n+k} \sum_{\alpha=1}^{p} \sum_{\alpha=p+1}^{n}\left(\mathbf{B}_{\alpha \beta}^{r}\right)^{2} \\
& +\sum_{\alpha=1}^{p} \sum_{\beta=p+1}^{n} g\left(R\left(e_{\alpha}, e_{\beta}\right) e_{\alpha}, e_{\beta}\right) .
\end{aligned}
$$

From $R\left(e_{\alpha}, e_{\beta}\right) e_{\alpha}=\frac{\mathcal{H}^{f}\left(e_{\alpha}, e_{\alpha}\right)}{f} e_{\beta}$ in (14), we derive

$$
\sum_{\alpha=1}^{p} \sum_{\beta=p+1}^{n} g\left(R\left(e_{\alpha}, e_{\beta}\right) e_{\alpha}, e_{\beta}\right)=\frac{q}{f} \sum_{\alpha=1}^{p} g\left(\nabla_{e_{\alpha}} \nabla f, e_{\alpha}\right) .
$$

Thus, from (26) and (27), we derive

$$
\begin{aligned}
\sum_{\alpha=1}^{p} \sum_{\beta=p+1}^{n}\left\{2\left\|\mathbf{B}\left(e_{\alpha}, e_{\beta}\right)\right\|^{2}-g\right. & \left.\left(\mathbf{B}\left(e_{\beta}, e_{\beta}\right), \mathbf{B}\left(e_{\alpha}, e_{\alpha}\right)\right)\right\} \\
= & \frac{q}{f} \sum_{\alpha=1}^{p} g\left(\nabla_{e_{\alpha}} \nabla f, e_{\alpha}\right)+\sum_{r=n+1}^{n+k} \sum_{\alpha=1}^{p} \sum_{\alpha=p+1}^{n}\left(\mathbf{B}_{\alpha \beta}^{r}\right)^{2} .
\end{aligned}
$$

Computing the Laplacian $\Delta f$, on $M^{n}$, one obtains:

$$
\begin{aligned}
\Delta f & =\sum_{i=1}^{n} g\left(\nabla_{e_{i}}(\nabla f), e_{i}\right) \\
& =\sum_{\alpha=1}^{p} g\left(\nabla_{e_{\alpha}}(\nabla f), e_{\alpha}\right)+\sum_{\beta=p+1}^{n} g\left(\nabla_{e_{\beta}}(\nabla f), e_{\beta}\right) .
\end{aligned}
$$


From the hypothesis of a warped product submanifold, $N_{1}^{p}$ is totally geodesic in $M^{n}$. It implies that gradf $\in \mathfrak{X}\left(T N_{1}\right)$, and from Lemma 1 (i)-(ii), we obtain

$$
\Delta f=\frac{1}{f} \sum_{j=p+1}^{n} g\left(e_{j}, e_{j}\right)\|\nabla f\|^{2}+\sum_{\alpha=1}^{p} g\left(\nabla_{e_{\alpha}}(\nabla f), e_{\alpha}\right) .
$$

Multiply the above equation by $\frac{1}{f}$, we get:

$$
\frac{\Delta f}{f}=\frac{1}{f} \sum_{\alpha=1}^{p} g\left(\nabla_{e_{\alpha}}(\nabla f), e_{\alpha}\right)+q\|\nabla(\ln f)\|^{2} .
$$

After some computations, we find that

$$
\frac{q}{f} \sum_{\alpha=1}^{p} g\left(\nabla_{e_{\alpha}}(\nabla f), e_{\alpha}\right)=\frac{q \Delta f}{f}-q^{2}\|\nabla \ln f\|^{2} .
$$

Thus, from (28) and (29), one obtains:

$$
\begin{aligned}
\sum_{\alpha=1}^{p} \sum_{\beta=1}^{q}\left\{2\left\|\mathbf{B}\left(e_{\alpha}, e_{\beta}\right)\right\|^{2}-g\right. & \left.\left(\mathbf{B}\left(e_{\beta}, e_{\beta}\right), \mathbf{B}\left(e_{\alpha}, e_{\alpha}\right)\right)\right\} \\
= & \sum_{r=n+1}^{n+k} \sum_{\alpha=1}^{p} \sum_{\beta=1}^{q}\left(\mathbf{B}_{\alpha \beta}^{r}\right)^{2}+\frac{q \Delta f}{f}-q^{2}\|\nabla \ln f\|^{2} .
\end{aligned}
$$

Using the Gauss Equation (5) for Euclidean space $\mathbb{E}^{n+k}$, we find that

$$
n^{2}\|\mathbf{H}\|^{2}=\|\mathbf{B}\|^{2}+\sum_{1 \leq A<B \leq n} K\left(e_{A} \wedge e_{B}\right) .
$$

The above equation can be written for the warped product manifold $\mathbb{M}^{n}$ and from the viewpoint of (6) and (14) as follows:

$$
\begin{aligned}
\sum_{r=n+1}^{n+k}\left(\sum_{A=1}^{n} \mathbf{B}_{A A}^{r}\right)^{2}= & \sum_{r=n+1}^{n+k} \sum_{i, j=1}^{p}\left(\mathbf{B}_{i j}^{r}\right)^{2}+\sum_{r=n+1}^{n+k} \sum_{a, b=p+1}^{n}\left(\mathbf{B}_{a b}^{r}\right)^{2} \\
& +2 \sum_{r=n+1}^{n+k} \sum_{\alpha=1}^{p} \sum_{\beta=p+1}^{n}\left(\mathbf{B}_{\alpha \beta}^{r}\right)^{2}+\sum_{\alpha=1}^{p} \sum_{\beta=p+1}^{n} K\left(e_{\alpha} \wedge e_{\beta}\right) \\
& +\sum_{1 \leq i<j \leq p} K\left(e_{i} \wedge e_{j}\right)+\sum_{1 \leq a<b \leq q} K\left(e_{a} \wedge e_{b}\right) .
\end{aligned}
$$

Using (11) and (19) in the above equation, we derive

$$
\begin{aligned}
\sum_{r=n+1}^{n+k}\left(\sum_{A=1}^{n} \mathbf{B}_{A A}^{r}\right)^{2} & =\sum_{r=n+1}^{n+k} \sum_{i, j=1}^{p}\left(\mathbf{B}_{i j}^{r}\right)^{2}+\sum_{r=n+1}^{n+k} \sum_{a, b=p+1}^{n}\left(\mathbf{B}_{a b}^{r}\right)^{2} \\
& +2 \sum_{r=n+1}^{n+k} \sum_{\alpha=1}^{p} \sum_{\beta=p+1}^{n}\left(\mathbf{B}_{\alpha \beta}^{r}\right)^{2}+\frac{q \Delta f}{f} \\
& +\sum_{1 \leq i<j \leq p} \widetilde{K}\left(e_{i} \wedge e_{j}\right)+\sum_{p+1 \leq a<b \leq n} \widetilde{K}\left(e_{a} \wedge e_{b}\right) \\
& +\sum_{r=n+1}^{n+k} \sum_{1 \leq i<j \leq p}\left(\mathbf{B}_{i i}^{r} \mathbf{B}_{j j}^{r}-\left(\mathbf{B}_{i j}^{r}\right)^{2}\right) \\
& +\sum_{r=n+1}^{n+k} \sum_{p+1 \leq a<b \leq n}\left(\mathbf{B}_{a a}^{r} \mathbf{B}_{b b}^{r}-\left(\mathbf{B}_{a b}^{r}\right)^{2}\right) .
\end{aligned}
$$


Thus, using the flatness of the space $\mathbb{E}^{n+k}$ and making some rearrangements in the last equation, one obtains:

$$
\begin{aligned}
\sum_{r=n+1}^{n+k}\left(\sum_{A=1}^{n} \mathbf{B}_{A A}^{r}\right)^{2}= & \sum_{r=n+1}^{n+k} \sum_{i, j=1}^{p}\left(\mathbf{B}_{i j}^{r}\right)^{2}+\sum_{r=n+1}^{n+k} \sum_{a, b=p+1}^{n}\left(\mathbf{B}_{a b}^{r}\right)^{2} \\
& +2 \sum_{r=n+1}^{n+k} \sum_{\alpha=1}^{p} \sum_{\beta=p+1}^{n}\left(\mathbf{B}_{\alpha \beta}^{r}\right)^{2}+\frac{q \Delta f}{f}-\sum_{r=n+1}^{n+k} \sum_{1 \leq i<j \leq p}\left(\mathbf{B}_{i j}^{r}\right)^{2} \\
& +\sum_{r=n+1}^{n+k} \sum_{1 \leq i<j \leq p} \mathbf{B}_{i i}^{r} \mathbf{B}_{j j}^{r}+\sum_{r=n+1}^{n+k}\left(\left(\mathbf{B}_{i i}^{r}\right)^{2}+\cdots+\left(\mathbf{B}_{p p}^{r}\right)^{2}\right) \\
& -\sum_{r=n+1}^{n+k}\left(\left(\mathbf{B}_{i i}^{r}\right)^{2}+\cdots+\left(\mathbf{B}_{p p}^{r}\right)^{2}\right)+\sum_{r=n+1}^{n+k} \sum_{p+1 \leq a<b \leq n} \mathbf{B}_{a a}^{r} h_{b b}^{r} \\
& -\sum_{r=n+1}^{n+k} \sum_{p+1 \leq a<b \leq n}\left(\mathbf{B}_{a a}^{r}\right)^{2}+\sum_{r=n+1}^{n+k}\left(\left(\mathbf{B}_{p+1 p+1}^{r}\right)^{2}+\cdots+\left(\mathbf{B}_{n n}^{r}\right)^{2}\right) \\
& -\sum_{r=n+1}^{n+k}\left(\left(\mathbf{B}_{p+1 p+1}^{r}\right)^{2}+\cdots+\left(\mathbf{B}_{n n}^{r}\right)^{2}\right) .
\end{aligned}
$$

After some rearrangements of the above equation, we get

$$
\begin{aligned}
\sum_{r=n+1}^{n+k}\left(\sum_{A=1}^{n} \mathbf{B}_{A A}^{r}\right)^{2} & =\sum_{r=n+1}^{n+k} \sum_{i, j=1}^{p}\left(\mathbf{B}_{i j}^{r}\right)^{2}+\sum_{r=n+1}^{n+k} \sum_{a, b=p+1}^{n}\left(\mathbf{B}_{a b}^{r}\right)^{2} \\
& +2 \sum_{r=n+1}^{n+k} \sum_{\alpha=1}^{p} \sum_{\beta=p+1}^{n}\left(\mathbf{B}_{\alpha \beta}^{r}\right)^{2}+\frac{q \Delta f}{f} \\
& +\sum_{r=n+1}^{n+k}\left\{\sum_{1 \leq i<j \leq p} \mathbf{B}_{i i}^{r} \mathbf{B}_{j j}^{r}+\left(\mathbf{B}_{11}^{r}\right)^{2}+\cdots+\left(\mathbf{B}_{p p}^{r}\right)^{2}\right\} \\
& -\sum_{r=n+1}^{n+k}\left\{\sum_{1 \leq i<j \leq p}\left(\mathbf{B}_{i j}^{r}\right)^{2}+\left(\mathbf{B}_{11}^{r}\right)^{2}+\cdots+\left(\mathbf{B}_{p p}^{r}\right)^{2}\right\} \\
& +\sum_{r=n+1}^{n+k}\left\{\sum_{p+1 \leq a<b \leq n} \mathbf{B}_{a a}^{r} \mathbf{B}_{b b}^{r}+\left(\mathbf{B}_{p+1 p+1}^{r}\right)^{2}+\cdots+\left(\mathbf{B}_{n n}^{r}\right)^{2}\right\} \\
& -\sum_{r=n+1}^{n+k}\left\{\sum_{p+1 \leq a<b \leq n}\left(\mathbf{B}_{a b}^{r}\right)^{2}+\left(\mathbf{B}_{p+1 p+1}^{r}\right)^{2}+\cdots+\left(\mathbf{B}_{n n}^{r}\right)^{2}\right\} .
\end{aligned}
$$

Using the binomial theorem and the fact that the base manifold $N_{1}^{p}$ of a warped product manifold $N_{1}^{p} \times_{f} N_{2}^{q}$ is minimal, then it is not hard to check that: 


$$
\begin{aligned}
\sum_{r=n+1}^{n+k}\left(\sum_{A=p+1}^{n} \mathbf{B}_{A A}^{r}\right)^{2}= & \sum_{r=n+1}^{n+k} \sum_{i, j=1}^{p}\left(\mathbf{B}_{i j}^{r}\right)^{2}+\sum_{r=n+1}^{n+k} \sum_{a, b=p+1}^{n}\left(\mathbf{B}_{a b}^{r}\right)^{2} \\
& +2 \sum_{r=n+1}^{n+k} \sum_{\alpha=1}^{p} \sum_{\beta=p+1}^{n}\left(\mathbf{B}_{\alpha \beta}^{r}\right)^{2}+\frac{q \Delta f}{f} \\
& +\sum_{r=n+1}^{n+k}\left(\left(\mathbf{B}_{i i}^{r}\right)^{2}+\cdots+\left(\mathbf{B}_{p p}^{r}\right)^{2}\right)-\sum_{r=n+1}^{n+k} \sum_{i, j=1}^{p}\left(\mathbf{B}_{i j}^{r}\right)^{2} \\
& +\sum_{r=n+1}^{n+k}\left(\left(\mathbf{B}_{p+1 p+1}^{r}\right)^{2}+\cdots+\left(\mathbf{B}_{n n}^{r}\right)^{2}\right) \\
& -\sum_{r=n+1}^{n+k} \sum_{a, b=p+1}^{n}\left(\mathbf{B}_{a b}^{r}\right)^{2} .
\end{aligned}
$$

Then, we obtain that:

$$
2 \sum_{r=n+1}^{n+k} \sum_{\alpha=1}^{p} \sum_{\beta=p+1}^{n}\left(\mathbf{B}_{\alpha \beta}^{r}\right)^{2}=-\frac{q \Delta f}{f} .
$$

From (30) and (37), we get:

$$
\begin{aligned}
\sum_{\alpha=1}^{p} \sum_{\beta=p+1}^{n}\left\{2\left\|\mathbf{B}\left(e_{\alpha}, e_{\beta}\right)\right\|^{2}-g\right. & \left(\mathbf{B}\left(e_{\beta}, e_{\beta}\right), \mathbf{B}\left(e_{\alpha}, e_{\alpha}\right)\right\} \\
= & \frac{q \Delta f}{f}-q^{2}\|\nabla \ln f\|^{2}-\frac{q \Delta f}{2 f},
\end{aligned}
$$

which is equivalent to

$$
\sum_{\alpha=1}^{p} \sum_{\beta=p+1}^{n}\left\{2\left\|\mathbf{B}\left(e_{\alpha}, e_{\beta}\right)\right\|^{2}-g\left(\mathbf{B}\left(e_{\beta}, e_{\beta}\right), \mathbf{B}\left(e_{\alpha}, e_{\alpha}\right)\right)\right\}=\frac{q \Delta f}{2 f}-q^{2}\|\nabla \ln f\|^{2} .
$$

From our assumption (20) and (38), we obtain

$$
\sum_{\alpha=1}^{p} \sum_{\beta=p+1}^{n}\left\{2\left\|\mathbf{B}\left(e_{\alpha}, e_{\beta}\right)\right\|^{2}-g\left(\mathbf{B}\left(e_{\beta}, e_{\beta}\right), \mathbf{B}\left(e_{\alpha}, e_{\alpha}\right)\right)\right\}<0 .
$$

Applying Theorem 1 for constant holomorphic section curvature $c=0$, we obtain that there are no stable $p$-currents in $M^{n}$ and $H_{p}\left(M^{n}, \mathbb{Z}=H_{q}\left(M^{n}, \mathbb{Z}\right)\right.$, which completes the proof of the first case of the Theorem.

In addition, if $n \geq 4$, we deduce that $H_{p}\left(M^{n}, \mathbb{Z}\right)=0$. Suppose that the fundamental group $\pi_{1}\left(M^{n}\right)$ is finite. From $H_{n-p}\left(M^{n}, \mathbb{Z}\right)=0=H_{q}\left(M^{n}, \mathbb{Z}\right)=0$ and the universal coefficient theorem, we infer that $H^{n-p}\left(M^{n}, \mathbb{Z}\right)$ has no torsion and neither does $H_{p}\left(M^{n}, \mathbb{Z}\right)$ by the Poincare duality. Because the $\pi_{1}\left(M^{n}\right)$ is finite, we have $H_{p}\left(M^{n}, \mathbb{Z}\right)=0$ and $H_{q}\left(M^{n}, \mathbb{Z}\right)=0$, then $M^{n}$ is the homology sphere. By considering the ambient manifold $\widetilde{M}$ of $M^{n \prime}$ 's Riemannian universal covering , the compactness of $M^{n}$, the Equation (39) and the Myer's theorem, we could obtain that $\widetilde{M}$ is compact. Because the $\widetilde{M}$ is a homology sphere, and the fundamental group is $\pi_{1}(\widetilde{M})=0$, and is the homotopy sphere as well, therefore applying the results from (Freedman $n=4$ [7], Smale $n \geq 5$ [27]), we get that $M^{n}$ is homotopic to the Euclidean sphere $\mathbb{S}^{n}$. As an immediate consequence of Sjerve [28], we get that the fundamental group $\pi_{1}\left(M^{n}\right)=0$, on $M^{n}$. This completes the second case of Theorem 3. 
From the case (i), there do not exist stable integral $p$-currents in a warped product submanifold $M^{n}$ and their homology groups are vanished, $H_{p}\left(M^{n}, \mathbb{Z}\right)=H_{q}\left(M^{n}, \mathbb{Z}\right)=0$, for all positive integer $p, q$ such that $p+q=n$. Moreover, Equation (39) shows that the Ricci curvature on $M^{n}$ is positive. Therefore, using the result of R. S. Hamilton [29] for $n=3$, we conclude that $M^{3}$ is a three dimensional spherical space form and hence $\pi_{1}(M)=0$, it is homotopic to a Euclidean sphere $\mathbb{S}^{3}$. This is the complete proof of Theorem 3 .

\subsection{Proof of Theorem 4}

If $f$ is a first eigenfunction of the Laplacian $\Delta f=\operatorname{div}(\nabla f)$ of $M^{n}$ associated to the first non zero eigenvalue $\lambda_{1}$, that is, $\Delta f=-\lambda_{1} f$, then we recall now Bochner formula (see, e.g., [30]), which states that for a differentiable function $f$ defined on a Riemannian manifold, the following relation holds:

$$
\frac{1}{2} \Delta\|\nabla f\|^{2}=\left\|\nabla^{2} f\right\|^{2}+\operatorname{Ric}(\nabla f, \nabla f)+g(\nabla f, \nabla(\Delta f)) .
$$

Integrating the above equation, using the Stoke's theorem, we get

$$
\int_{M^{n}}\left\|\nabla^{2} f\right\|^{2} d V+\int_{M^{n}} \operatorname{Ric}(\nabla f, \nabla f) d V+\int_{M^{n}} g(\nabla f, \nabla(\Delta f)) d V=0 .
$$

Now, using $\Delta f=-\lambda_{1} f$ and making some rearrangement in Equation (40), we derive

$$
\int_{M^{n}}\|\nabla f\|^{2} d V=\frac{1}{\lambda_{1}}\left(\int_{M^{n}}\left\|\nabla^{2} f\right\|^{2} d V+\int_{M^{n}} \operatorname{Ric}(\nabla f, \nabla f) d V\right) .
$$

As we assume that Equation (22) holds, that is

$$
\int_{M^{n}}\left\{\left\|\nabla^{2} f\right\|^{2}+\operatorname{Ric}(\nabla f, \nabla f)\right\} d V>\left(\frac{\lambda_{1}^{2}}{2 q}\right)\left(-\int_{M^{n}} f^{2} d V\right) .
$$

Combining the Equations (40) and (42), we get

$$
\int_{M^{n}}\|\nabla f\|^{2} d V>\frac{1}{\lambda_{1}}\left(\frac{\lambda_{1}^{2}}{2 q}\right)\left(-\int_{M^{n}} f^{2} d V\right),
$$

which is equivalent to the following:

$$
2 q \int_{M^{n}}\|\nabla f\|^{2} d V>-\int_{M^{n}} f\left(f \lambda_{1}\right) d V
$$

Using $\Delta f=-\lambda_{1} f$, we get

$$
2 q \int_{M^{n}}\|\nabla f\|^{2} d V>\int_{M^{n}} f \Delta f d V .
$$

Then condition (2) is replaced by the above pinching condition in Theorem 3 for a Euclidean space $\mathbb{E}^{p+q+k}$. Then, from the above equation and invoking Theorem 3 , we get the final conclusion of our theorem. This completes the proof of the theorem.

\subsection{Proof of Corollary 1}

As we know that $M^{n}$ is an $N_{1}^{P}$-minimal compact warped product submanifold, then from Gauss equation is given by:

$$
R_{j k l}^{i}=\delta_{i k} \delta_{j l}-\delta_{i l} \delta_{j k}+\sum_{r=1}^{p+q+k}\left(\mathbf{B}_{i k}^{r} \mathbf{B}_{j l}^{r}-\mathbf{B}_{i l}^{r} \mathbf{B}_{j k}^{r}\right),
$$


which implies the following:

$$
R_{j i j}^{i}=\delta_{i i} \delta_{j j}-\delta_{i j} \delta_{j i}+\sum_{r=1}^{p+q+k}\left(\mathbf{B}_{i i}^{r} \mathbf{B}_{j j}^{r}-\mathbf{B}_{i j}^{r} \mathbf{B}_{j i}^{r}\right)
$$

Taking into account that $N_{1}^{p}$ is a minimal submanifold and using the argument of the Ricci curvature for an Euclidean space, we get:

$$
\operatorname{Ric}\left(e_{i}, e_{j}\right)=-\sum_{r=1}^{p+q+k} \sum_{l=1}^{p} \mathbf{B}_{i l}^{r} \mathbf{B}_{j l}^{r}
$$

The above equation yields that

$$
\operatorname{Ric}\left(f_{i} e_{i}, e_{j} f_{j}\right)=-\sum_{r=1}^{p+q+k} \sum_{l=1}^{p} \mathbf{B}_{i l}^{r} \mathbf{B}_{j l}^{r} f_{i} f_{j}
$$

Taking the trace of Equation (47), we get

$$
\operatorname{Ric}(\nabla f, \nabla f)=-\sum_{i=1}^{p}\left\|\mathbf{B}\left(\nabla f, e_{i}\right)\right\|^{2} .
$$

Inserting the above equation into (21), we derive that

$$
\int_{M^{n}}\left\{\left\|\nabla^{2} f\right\|^{2}-\sum_{i=1}^{p}\left\|\mathbf{B}\left(\nabla f, e_{i}\right)\right\|^{2}\right\} d V>\left(\frac{1}{2 q}\right)\left(-\int_{M^{n}}\left(\lambda_{1} f\right)^{2} d V\right),
$$

which implies the following from the fact that $\Delta f=-\lambda_{1} f$ in the above equation:

$$
\int_{M^{n}}\left\{\left\|\nabla^{2} f\right\|^{2}-\sum_{i=1}^{p}\left\|\mathbf{B}\left(\nabla f, e_{i}\right)\right\|^{2}\right\} d V>\left(\frac{1}{2 q}\right)\left(-\int_{M^{n}}(\Delta f)^{2} d V\right) .
$$

Thus we obtain the required result (22). This completes the proof of the corollary.

\subsection{Proof of Corollary 2}

Using the hypothesis of corollary, $\nabla f \in \operatorname{Ker} \mathbf{B}$, which implies that $\mathbf{B}\left(\nabla f, e_{i}\right)=0$. Using this condition in (22), we can easily obtain the required result.

\subsection{Proof of Corollary 3}

As we assume that $M^{n}$ is Ricci-flat, then $\operatorname{Ric}(\nabla f, \nabla f)=0$. Using these conditions in Equation (43), we obtain required result.

\section{Conclusions}

When the base manifold is minimal in ambient manifolds, the results in this paper can be considered an extension of Xin's [2] work in the framework of compact warped product submanifolds. We proved a compact warped product submanifold $M^{n}$ in a Euclidean space $\mathbb{E}^{n+k}$, that there are no stable $p$-currents, homology groups vanish, and that $M^{3}$ is homotopic to the Euclidean sphere $\mathbb{S}^{3}$ under various extrinsic restrictions, involving the eigenvalue of the warped function, integral Ricci curvature and the Hessian tensor. In our next work, we will combine the singularity theory presented in [31-34] to study compact warped product submanifold $M^{n}$ in a Euclidean space $\mathbb{E}^{n+k}$.

Author Contributions: Conceptualization, Y.L., P.L.-I., A.A. and A.H.A.; methodology, Y.L., P.L.-I., A.A. and A.H.A.; writing-original draft preparation, Y.L., P.L.-I., A.A. and A.H.A.; writing-review and editing, Y.L., P.L.-I., A.A. and A.H.A. All authors have read and agreed to the published version of the manuscript. 
Funding: Research groups program under grant number R.G.P1/186/41, scientific research at King Khalid University.

Institutional Review Board Statement: Not applicable.

Informed Consent Statement: Not applicable.

Data Availability Statement: There is no data use for this manuscript.

Acknowledgments: The authors extend their appreciation to the deanship of scientific research at King Khalid University for funding this work through research groups program under grant number R.G.P1/186/41.

Conflicts of Interest: The authors declare no competing of interest.

\section{References}

1. Lawson, H.B.; Simons, J. On stable currents and their application to global problems in real and complex geometry. Ann. Math. 1973, 98, 427-450. [CrossRef]

2. Xin, Y.L. An application of integral currents to the vanishing theorems. Sci. China Ser. A. 1984, $27,233-241$.

3. Vlachos, T. The Ricci curvature of submanifolds and its applications. Quart. J. Math. 2003, 55, 225-230. [CrossRef]

4. Wallah, N.R. Minimal Immersion of Symmetric Spaces into Spheres; Symmetric Spaces, Dekker: New York, NY, USA, 1972; pp. 1-40.

5. Vlachos, T. Homology vanishing theorems for submanifolds. Proc. Amer. Math. Soc. 2007, 135, 2607-2617. [CrossRef]

6. Xu, H.W.; Gu, J.R. Geometric, topological and differentiable rigidity of submanifolds in space forms. Geom. Funct. Anal. 2013, 23, 1684-1703. [CrossRef]

7. Freedman, M. The topology of four-dimensional manifolds. J. Diff. Geom. 1982, 17, 357-453. [CrossRef]

8. Fu, H.P.; Xu, H.W. Vanishing and topological sphere theorems for submanifolds of Hyperbolic space. Intern. J. Math. 2008, 19, 811-822. [CrossRef]

9. Gauchman, H. Minimal submanifolds of sphere with bounded second fundamental form. Trans. Am. Math. Soc. 1993, 79, 779-791. [CrossRef]

10. Liu, L.; Zhang, Q. Non-existence of stable currents in submanifolds of the Euclidean spaces. J. Geom. 2009, 96, 125-133. [CrossRef]

11. Sahin, F. On the topology of CR-warped product submanifolds. Int. J. Geom. Meth. Mod. Phy. 2018, 15, 1850032. [CrossRef]

12. Shiohama, K.; Xu, H. The topological sphere theorem for complete submanifolds. Comp. Maths. 1997, 107, 221-232. [CrossRef]

13. Xu, H.W.; Ye, F. Differentiable sphere theorems for submanifolds of positive $k$-th ricci curvature. Manu. Math. 2012, 138, 529-543. [CrossRef]

14. Zhang, Z.X. Non-existence of stable currents in submanifolds of a product of two spheres. Bull. Austral. Math. Soc. 1991, 44, 325-336. [CrossRef]

15. Nash, J. The imbedding problem for Riemannian manifolds. Ann. Math. 1956, 63, 20-63. [CrossRef]

16. Nolker, S. Isometric immersions of warped products. Diff. Geom. Appl. 1996, 6, 1-30. [CrossRef]

17. Liaqat, M.; Laurian-Ioan, P.; Othman, W.A.M.; Ali, A.; Gani, A.; Ozel, C. Estimation of inequalities for warped product semi-slant submanifolds of Kenmotsu space forms. J. Inequal. Appl. 2016, 2016, 239. [CrossRef]

18. Alluhaibi, N.; Mofarreh, F.; Ali, A.; Mior Othman, W.A. Geometric inequalities of warped product submanifolds and their applications. Mathematics 2020, 8, 759. [CrossRef]

19. Bishop, R.L.; O’Neil, B. Manifolds of negative curvature. Trans. Amer. Math. Soc. 1969, 145, 1-9. [CrossRef]

20. Chen, B.Y. On isometric minimal immersions from warped products into real space forms. Proc. Edinb. Math. Soc. 2002, 45, 579-587. [CrossRef]

21. Ali, A.; Mofarreh, F.; Ozel, C.; Othman, W.A.M. Homology of warped product submanifolds in the unit sphere and its applications. Int. J. Geom. Meth. Mod. Phys. 2020, 17, 2050121. [CrossRef]

22. Kenna, R. Homotopy in statistical physics. Condens. Matter Phys. 2006, 9, 283-304. [CrossRef]

23. Li, Y.L.; Lone, M.A.; Wani, U.A. Biharmonic submanifolds of Kaehler product manifolds. AIMS Math. 2021, 6, 9309-9321. [CrossRef]

24. Li, Y.L.; Ali, A.; Ali, R. A general inequality for CR-warped products in generalized Sasakian space form and its applications. Adv. Math. Phys. 2021, 2021, 5777554. [CrossRef]

25. Major, S.; Rideout, D.; Surya, S. Stable homology as an indicator of manifoldlikeness in causal set topology. Class. Quantum Grav. 2009, 26, 175008. [CrossRef]

26. Surya, S. Causal set topology. Theor. Comput. Sci. 2008, 405, 188-197. [CrossRef]

27. Smale, S. Generalized Poincare's conjecture in dimensions greater than four. Ann. Math. 1961, 74, 391-406. [CrossRef]

28. Sjerve, D. Homology spheres which are covered by spheres. J. Lond. Math. Soc. 1973, 6, 333-336. [CrossRef]

29. Hamilton, R.S. Three-manifolds with positive Ricci curvature. J. Diff. Geom. 1982, 17, 255-306. [CrossRef]

30. Berger, M.; Gauduchon, P.; Mazet, E. Le spectre d'une variété Riemannienne; Springer: Berlin/Heidelberg, Germany, 1971; Volume 194, pp. 141-241. 
31. Li, Y.L.; Liu, S.Y; Wang, Z.G. Tangent developables and Darboux developables of framed curves. Topology Appl. 2020, 107526. [CrossRef]

32. Li, Y.L.; Wang, Z.G. Lightlike tangent developables in de Sitter 3-space. J. Geom. Phys. 2021, 164, 104188. [CrossRef]

33. Li, Y.L.; Wang, Z.G.; Zhao, T.H. Geometric Algebra of Singular Ruled Surfaces. Adv. Appl. Clifford Algebras. 2021, 31, 19. [CrossRef]

34. Li, Y.L.; Zhu, Y.S.; Sun, Q.Y. Singularities and dualities of pedal curves in pseudo-hyperbolic and de Sitter space. Int. J. Geom. Meth. Mod. Phys. 2021, 18, 2150008-68. [CrossRef] 